\title{
Flujo en un canal interbahías para la renovación de las aguas en Bahía San Vicente (Chile)
}

\author{
Flow in an interbay channel for water renovation in San Vicente Bay (Chile)
}

Fecha de entrega: 31 de agosto 2021 Fecha de aceptación: 24 de noviembre 2021

\section{María Pedreros-Guarda ${ }^{1}$ y Dante Figueroa ${ }^{2}$}

${ }^{1}$ Departamento de Sistemas Acuáticos, Facultad de Ciencias Ambientales, Universidad de Concepción, Concepción, Chile, mapedreros@udec.cl

2 Departamento de Geofísica, Facultad de Ciencias Físicas y Matemáticas, Universidad de Concepción, Concepción, Chile, dantefigueroa@udec.cl

La bahía San Vicente, en el litoral de Chile central, es un cuerpo de agua particularmente contaminado. En este trabajo se propone la construcción de un canal que la comunique con la bahía de Concepción, para ayudar a su descontaminación. Usando una versión corregida del modelo de canal de Vennell se calcula el flujo de agua entre ambas bahias, sin y con una compuerta entre ellas, y se muestra que en el segundo caso la combinación de diferencia mareal y los vientos logran en cerca de un mes la renovación de las aguas de la zona más críticamente contaminada de bahía San Vicente.

Palabras clave: canal artificial, forzante mareal, bahía contaminada, Chile Central
San Vicente Bay, in central Chile, is a particularly polluted body of water. In this work the construction of a canal that connects it with Concepción Bay is proposed, to help decontaminate it. Using a modified version of the Vennell channel model, the water flow between the two bays is calculated, without and with a gate between them. It is shown that in the second case the combination of the tidal difference and the winds achieved in about a month the renovation of the most critical part of San Vicente Bay.

Keywords: artificialchannel, tidalforcing, contaminated bay, Central Chile

\section{Introducción}

Las bahías son zonas marinas protegidas de manera natural, por lo que muchos grupos humanos se concentran en su entorno (DIRECTEMAR, 2019). En consecuencia allí se desarrollan actividades comerciales, estratégicas, y recreacionales. Este uso trae consigo grandes descargas de sustancias provenientes de desechos industriales y/o urbanos, eventuales derrames de combustibles asociados a actividades de puerto, además de obras de ingeniería que aumentan los tiempos de residencia del agua, todo lo cual somete a las bahías a una alta presión ambiental (Valenzuela, 2003; Rudolph et al., 2002).

En la región del Biobío (Chile) se encuentra el caso de una zona fuertemente alterada debido a actividades humanas: las bahías de San Vicente y Concepción (Aguirre-Martínez et al., 2009, ver Figura 1). Ellas están separadas por la comuna de Talcahuano, la que, a partir de 1950, tuvo un importante crecimiento industrial, pesquero, militar y portuario, que generó posteriormente un acelerado crecimiento urbano (Valenzuela, 2003).

La Bahía San Vicente, aunque relativamente pequeña, concentra una gran cantidad de usos de borde costero en su parte norte (DIRECTEMAR, 2019). La capacidad de asimilación del agua se ha visto sobrepasada por la descarga local de desechos (Rudolph et al., 2002). Además, se construyó un rompeolas, también en la zona norte, que aumentó el tiempo de residencia del agua en la bahía (Ahumada et al., 1989). Y lo anterior se exacerba aún más por una circulación marina dominante en sentido antihorario que mueve agua hacia el norte de Bahía San Vicente (Ahumada et al., 1989). El sector portuario e industrial comprende alrededor del $15 \%$ de la superficie de la bahía y presenta un alto grado de deterioro ambiental (Rudolph et al., 2002). 
a)

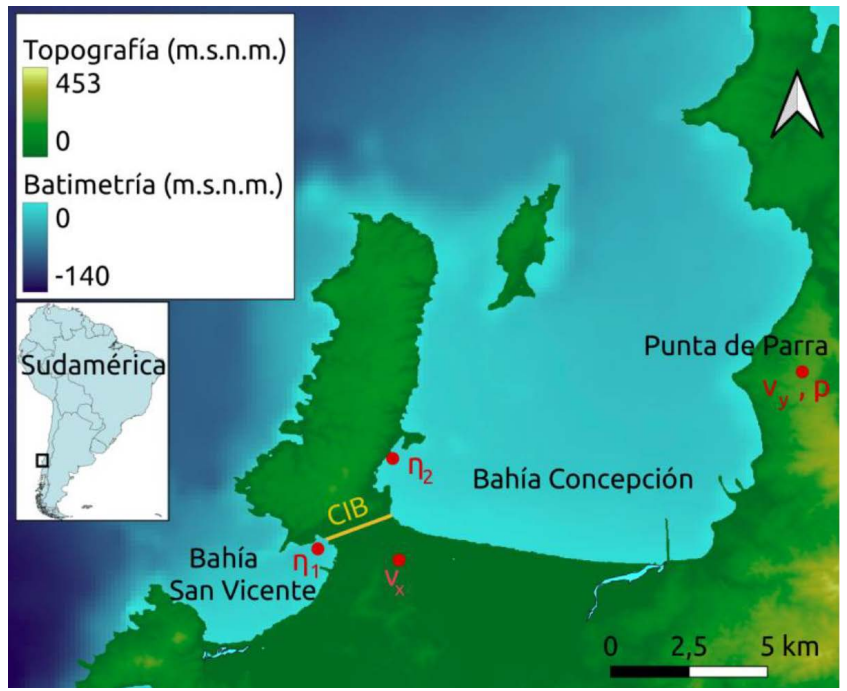

b)

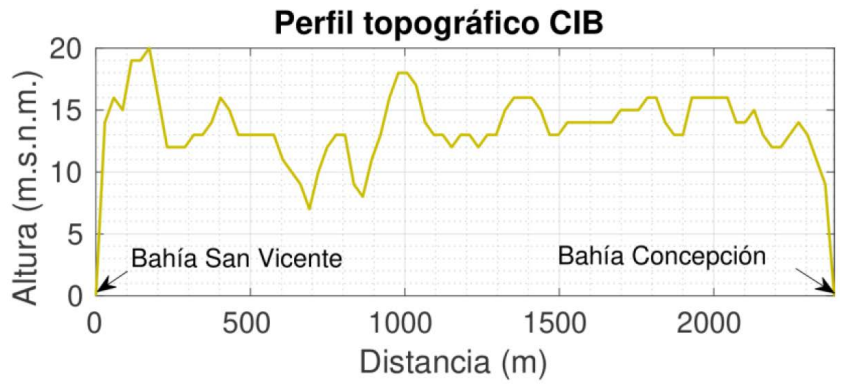

Figura 1: a) Área de estudio, bahías San Vicente y Concepción, y Canal Interbahías (CIB, línea amarilla). Los puntos rojos señalan las posiciones de las estaciones de datos usados en este trabajo (ver Tablas 1 y 3) y b) perfil topográfico CIB, construido a partir del modelo de elevación digital NASA SRTM 30m (Earth Engine Data Catalog, https://developers.google.com/earth engine/datasets/catalog/USGS_SRTMGL1_003 (29/08/2021))

En este trabajo se plantea una propuesta para fomentar la renovación de las aguas de la Bahía San Vicente, por medio de un canal navegable que una esta bahía con la vecina bahía de Concepción. Este canal, que en el presente estudio será denominado Canal Interbahías (CIB), transportaría agua desde la bahía de Concepción a la de San Vicente, ayudando a diluir las aguas de esta última. Adicionalmente, el Canal Interbahías permitiría el paso de navegaciones menores, acortando significativamente el tiempo de traslado entre ambas bahías, y fomentaría el turismo local.

La ubicación del CIB fue escogida considerando las zonas de mínima distancia entre ambas bahías, evitando zonas de relevancia ecológica (humedales, zonas protegidas), y escogiendo zonas de menor densidad urbana. Contemplando todo lo anterior, el CIB cruzaría la línea férrea y siete carreteras, y ocuparía el sitio de 84 casas y 12 bodegas. Se sugiere que sus dimensiones sean $2.4 \mathrm{~km}$ de largo, $10 \mathrm{~m}$ de ancho, y $3 \mathrm{~m}$ de profundidad. Dado que este es un trabajo exploratorio para confirmar la viabilidad física de este canal, sus dimensiones espaciales fueron elegidas para producir la mínima alteración posible al territorio y paisaje, pero cuidando de que éste sea útil para naves menores, las que usualmente deben hacer la peligrosa ruta bordeando península de Tumbes. Las dimensiones del canal permitirán la circulación de kayaks, botes, lanchas menores (eslora hasta $12 \mathrm{~m}$ ) y lanchas medianas (esloras hasta $15 \mathrm{~m})$.

Desde hace mucho tiempo existen canales de navegación artificiales entre cuerpos de agua oceánicos, usualmente con propósitos de navegación. Algunos ejemplos son los canales de Corinto (1893), de Suez (1869), y de Kiel (1895). Su objetivo es permitir el paso de navegaciones de turismo y/o comercio.

Los mencionados canales de Corinto, Suez y Kiel son, comparados con el que se propone en este estudio, relativamente grandes. Sus dimensiones van desde los 8 a $23 \mathrm{~m}$ de profundidad, 21 a $345 \mathrm{~m}$ de ancho y 6 a $193 \mathrm{~km}$ de largo. Gracias a estos canales es posible ahorrar hasta $8900 \mathrm{~km}$ de distancia de navegación. En tanto, el CIB es relativamente pequeño, y permitiría ahorrar unos $40 \mathrm{~km}$ de navegación entre ambas bahías, es decir, reduciría la distancia de navegación al 5\% de la actual, disminuyendo significativamente, además, el riesgo del viaje.

Físicamente, la idea del CIB se sustenta en el hecho de que una onda de marea se propaga de tal manera que llega antes a una bahía que a otra (Blanchfield et al., 2008). El desfase en la onda mareal provoca una diferencia de nivel del mar entre ambas bahías. Si existiera el CIB, este desnivel induciría un flujo a lo largo del canal (Garret y Cummins, 2005). Así es como funcionan los canales mareales en general (Garrett y Cummins, 2005; Blanchfield et al., 2008). Vennell $(2010,2011)$ propone un modelo que describe el flujo a través de un canal mareal que conecta dos cuerpos de agua. Basándose en este modelo, se implementa aquí un modelo para calcular el flujo que habría en el CIB. Dado que en distintos momentos del ciclo de marea el flujo puede ir hacia cualquiera de las dos bahías, se estudia el caso de un canal con una compuerta. Así, el flujo sería bloqueado cuando vaya hacia la bahía Concepción. El bloqueo sería en base a la onda de marea, en primera instancia. El objetivo de este trabajo es caracterizar el flujo que circularía en el CIB considerando distintos escenarios de viento y marea. 
El presente trabajo es un esfuerzo preliminar para evaluar si los forzantes diferencia de nivel del mar, vientos, y fricción con paredes y fondo posibilitan el transporte de agua en cantidades suficientes como para ventilar en forma significativa la Bahía San Vicente. Por su naturaleza exploratoria del efecto de los forzantes físicos, en el trabajo no se considera los impactos medioambientales ni territoriales de este canal, aspectos que serán evaluados en siguientes etapas, una vez demostrada la viabilidad del transporte de agua.

\section{Área de estudio}

El área de estudio comprende las bahías Concepción y San Vicente, y el Canal Interbahías (ver Figura 1), región del Biobío, Chile. La Bahía San Vicente tiene una superficie de $17.4 \mathrm{~km}^{2}$, un volumen de aproximadamente 278.4 x $10 \square \mathrm{m}^{3}$, una profundidad promedio de $16 \mathrm{~m}$ (Rudolph et al., 2002) y un tiempo de residencia de sus aguas de 20 horas (Ahumada, 1992). Es una bahía relativamente pequeña, abierta hacia el oeste. La Bahía Concepción, en tanto, tiene una superficie de $190 \mathrm{~km}^{2}$ (Srain y Rudolph, 2010) y un tiempo de residencia de sus aguas cercano a 3 días (Ahumada et al., 1983). Es una bahía rectangular y somera, con una profundidad máxima de $48 \mathrm{~m}$ (Sobarzo et al., 1997), abierta hacia el norte. Esta orientación la deja expuesta a los vientos del norte, lo que hace que sea propensa a acumular agua en su cabeza en un escenario de viento norte, debido a la transferencia de momento desde el viento hacia el agua.

El comportamiento de la presión atmosférica a lo largo del año resulta del efecto del anticiclón del Pacífico Sur, que en general produce vientos del suroeste, y eventos de escala sinóptica, asociados a mal tiempo y vientos norte, cuya duración es del orden de algunos días (Falvey y Garreaud, 2007). Como se trata de un canal somero (3 m de profundidad), el efecto del arrastre del viento sobre el flujo podría ser importante, razón por la cual se lo incluye en el modelo de flujo en canales de la sección Modelo numérico, adicionalmente a los forzantes considerados por Vennell $(2010,2011)$.

\section{Datos y métodos Modelo numérico}

Se toma como base el modelo unidimensional de flujo en canales de marea propuesto por Vennell $(2010,2011)$, y se le añade el efecto del arrastre del viento (Vallis, 2006). El modelo es un balance de momentum y se muestra en la ecuación (1). El cambio en la velocidad del flujo, $u$, es inducido por tres términos o tres forzantes, que de izquierda a derecha son: desnivel del mar, roce de fondo, y arrastre del viento. El modelo asume que la sección transversal $(A)$ del canal es rectangular y que la velocidad es constante tanto a lo largo del canal $(L)$ como en su sección transversal. Así, la velocidad, $u$, se relaciona con el transporte, $U$, mediante $U=u A$, donde $A=W h$. Debido a que es un modelo unidimensional, la velocidad no cambia ni en el eje $x$ (a lo largo de $L$ ) ni en el eje $z$ (porque no cambia en $A$ ). Es decir, $\partial u / \partial x=0$ y $\partial u / \partial z=0$, dejando como única variable independiente al tiempo (ecuación (1)). El equipo de Vennell ha venido desarrollando modelos conceptuales simples de canales de marea (por ejemplo, ver Vennell $(2010,2011)$ ), los que capturan los principales aspectos hidrodinámicos de esos canales. Una aplicación del modelo de Vennell a la circulación marina en el Canal de Chacao mostró resultados muy semejantes a los obtenidos con un modelo numérico 3D completo (Pedreros-Guarda, 2020). Las suposiciones de que gradientes longitudinales y verticales en el nivel del mar no aportan significativamente al flujo promedio del canal se ven de esta forma respaldadas.

$$
\frac{\partial u}{\partial t}=-\frac{g}{L}(\Delta \eta)-\frac{C_{D}}{h}|u| u+\frac{\tau^{x}}{h \rho}
$$

donde $u$ es la velocidad en el canal, $L$ es el largo del canal, $h$ la profundidad del canal, $\rho$ densidad del agua de mar, $\Delta \eta$ desnivel del mar entre los extremos del canal, $C_{D}$ el coeficiente de arrastre debido a fricción de fondo y lateral, y $\tau^{x}$ el esfuerzo de la componente del viento paralela al canal, el cual se puede calcular con la ecuación (2) (Vallis, 2006):

$$
\tau^{x}=\frac{C_{D V}\left|v_{x}\right| v_{x} \rho_{a i r}}{\rho}
$$

donde $C_{D V}$ es el coeficiente de arrastre del viento (adimensional), $\rho_{\text {air }}$ es la densidad del aire que se supuso constante en este estudio, con valor $1.24 \mathrm{~kg} / \mathrm{m}^{3}$ (Tabla 2), y $v_{x}$ es la velocidad del viento en la componente paralela al canal (Vallis, 2006). La fricción entre el agua y el canal se representó en este estudio mediante una fuerza viscosa 
turbulenta, cuadrática, como se muestra en la ecuación (3). Para el coeficiente de deriva, $C_{D}$, se usó el valor 0.0038 , dado que este valor de $C_{D}$ corresponde a un número de Manning de 0.012, que, según literatura, representa el caso de concreto emparejado con llana (Chow, 1994).

$$
F_{C_{D}}=\frac{1}{2} \rho A C_{D}|u| u
$$

\section{Solución numérica del modelo}

El modelo (ecuación (1)) fue resuelto mediante el método de diferencias finitas (Solorza et al., 2010) obteniéndose la ecuación (4):

$$
u_{k+1}=u_{k}+\Delta t_{k}\left(\frac{\partial u}{\partial t}\right)_{k}+O\left(\Delta t^{2}\right)
$$

donde $k$ es la posición $k$-ésima de un vector (por ejemplo: $u$, $t$ o $\partial U / \partial t), \Delta t$ es el intervalo de tiempo entre dos posiciones $k$ y $k+1$ de un vector y $O\left(\Delta t^{2}\right)$ es el error de representar al diferencial parcial por la ecuación de diferencias.

Lo que se hace con la ecuación (4) es integrar numéricamente en el tiempo mediante diferencias finitas. Como el modelo asume que no hay variaciones espaciales de la velocidad, tanto ella como sus forzantes (desnivel del mar, arrastre del viento y roce de fondo) variarán únicamente en función del tiempo. En la ecuación (4) se puede observar que para construir el elemento $u_{k+1}$ se requiere el elemento $u_{k}$. Esto obliga a imponer una condición inicial $u_{0}=0$, a falta de un valor existente. Lo mismo aplica para $U$, siendo la condición inicial $U_{0}=0$.

Reemplazando la ecuación (1) en la ecuación (4) y despreciando el error $O\left(\Delta t^{2}\right)$, se obtiene la solución al modelo numérico (sección Modelo numérico) en la ecuación (5). Además, considerando que la velocidad, $u \mathrm{y}$ el transporte, $U$, se relacionan mediante $U=u A$ (sección Modelo numérico) se obtiene también la ecuación (6).

$$
\begin{aligned}
& u_{k+1}=u_{k}+\Delta t_{k}\left(-\frac{g}{L}(\Delta \eta)_{k}-\frac{c_{D}}{h}\left|u_{k}\right| u_{k}+\frac{\tau_{k}^{x}}{h \rho}\right) \\
& U_{k+1}=U_{k}+\Delta t_{k}\left(-\frac{g A}{L}(\Delta \eta)_{k}-\frac{C_{D}}{h A}\left|U_{k}\right| U_{k}+W \frac{\tau_{k}^{x}}{\rho}\right)
\end{aligned}
$$

\section{Datos de entrada del modelo}

Los datos de entrada del modelo (ecuaciones (5) y (6)) se resumen en las Tablas 1 y 2. Los datos de nivel del mar usados para comparar las bahías de Concepción y San Vicente (ver Tabla 1) corresponden a 37 días, que es el único período de tiempo que a la fecha dispone el Centro Nacional de Datos Oceanográficos de Chile (CENDOC) para mediciones de nivel del mar simultáneas en ambas bahías. El desnivel del mar se construye a partir del nivel del mar en Bahía San Vicente $\left(\eta_{1}\right)$ y Bahía Concepción $\left(\eta_{2}\right)$ en la forma: $\Delta \eta=\eta_{2}-\eta_{1}$. El desnivel del mar puede verse en la Figura 2.

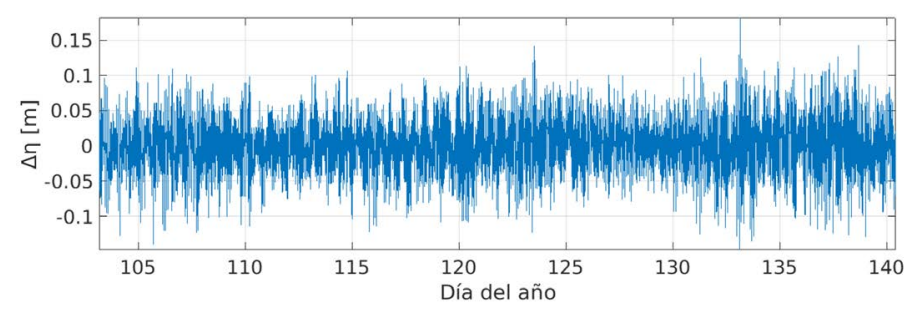

Figura 2: Forzante desnivel del mar $\Delta \eta$ como función del tiempo en el CIB para el período del 13/04/2010 al 20/05/2010 (Tabla 1)

Tabla 1: Datos usados en el modelo numérico (ecuación (5)), período, intervalo de tiempo entre dos posiciones $k$ de un vector

\begin{tabular}{|c|c|c|c|}
\hline Variable (Figura 1) & Período & $\Delta t$ & Fuente \\
\hline$\eta_{1}, \eta_{2}$ & \multirow{2}{*}{$\begin{array}{c}13 / 04 / 2010 \mathrm{a} \\
20 / 05 / 2010\end{array}$} & \multirow{2}{*}{$300 \mathrm{~s}^{*}$} & SHOA \\
\hline$v_{x}$ & & & INPESCA \\
\hline
\end{tabular}
y fuente de los datos: Servicio Hidrográfico y Oceanográfico de la Armada (SHOA) e Instituto de Investigación Pesquera (INPESCA).

*La componente $v_{x}$ del viento originalmente estaba en intervalos de tiempo de 1 hora, pero fue interpolada.

Tabla 2: Parámetros usados en el modelo numérico (ecuación (5)).

\begin{tabular}{|c|c|c|}
\hline Parámetro & Valor & Fuente \\
\hline$C_{D}$ & 0.0038 & $\begin{array}{c}\text { Sección Modelo numérico; } \\
\text { Vennell (2011); Wu } \text { et al. } \\
\text { (1999) }\end{array}$ \\
\hline$C_{D V}$ & $10^{-3}$ (valor típico) & Trenberth et al. (1989) \\
\hline$\rho_{\text {air }}, \mathrm{kg} / \mathrm{m}^{3}$ & 1.24 & \multirow{2}{*}{} \\
\hline$\rho, \mathrm{kg} / \mathrm{m}^{3}$ & 1025 & \\
\hline$g, \mathrm{~m} / \mathrm{s}^{2}$ & 9.81 & \\
\hline$L, \mathrm{~m}$ & 2400 & \\
\hline$W, \mathrm{~m}$ & 10 & \\
\hline$h, \mathrm{~m}$ & 3 & \\
\hline
\end{tabular}

Es importante revisar el intervalo de tiempo $(\Delta t)$ entre los datos de las series $\Delta \eta$ y $\tau^{x}$, pues debido a la condición de Courant-Friedrichs-Lewy (condición CFL(Russell, 1989)), 
la velocidad máxima que arroje el modelo $\left(u_{\max }\right)$ debe ser menor que la que permiten los parámetros $L \mathrm{y} \Delta t$ elegidos. Así, dado un cierto largo del canal, esta condición impone un cierto intervalo de tiempo $(\Delta t)$. A partir del número de CFL (Russell, 1989) se obtiene que el intervalo de tiempo máximo posible es:

$$
\Delta t=\frac{L}{u_{\max }}
$$

Si los vectores $\left(\Delta t \mathrm{y} \tau^{x}\right)$ tienen originalmente un $\Delta t$ mayor, entonces se debe interpolar.

\section{Efecto del viento en el desnivel del mar}

El forzante desnivel del mar en el modelo (sección Modelo numérico) es producido por la marea. Sin embargo, existe un efecto mecánico del viento que puede producir la acumulación o extracción del agua en los extremos del canal. Esto alteraría el volumen de alguna de las bahías, cambiando el forzante desnivel del mar. En el caso particular de la Bahía Concepción (uno de los extremos del canal) es sabido que la componente norte-sur del viento acumula o saca agua de la bahía, alterando su volumen (Sobarzo et al., 1997). En el caso de la bahía San Vicente, este efecto no ha sido reportado.

Bahía San Vicente es una bahía abierta y pequeña. Su área es el 11\% de Bahía Concepción y el tiempo de renovación de sus aguas la tercera parte (ver sección Área de estudio). Por ello se desprecia el efecto del viento en el volumen de la Bahía San Vicente. Es decir, se asume que su nivel del mar rápidamente se ajusta al del océano abierto. Luego, el forzante desnivel del mar es alterado únicamente por el efecto del viento en el volumen de la Bahía Concepción.

Con el objetivo de cuantificar el efecto de acumulación de agua en la Bahía Concepción se comparan el nivel del mar residual $\left(\eta_{r}\right)$ y la componente norte-sur del viento de eventos de 2 a 5 días de duración $\left(v_{y}\right)$. Con ambas series de tiempo se encuentra la relación entre la rapidez del viento y el cambio en el nivel del mar en la cabeza de Bahía Concepción. Con esta relación se construye el forzante desnivel del mar bajo diferentes condiciones de viento, con lo cual se puede calcular este efecto en el flujo en el CIB. El nivel del mar residual $\left(\eta_{r}\right)$ es aquel al que se le ha descontado la componente mareal $\left(\eta_{m}\right)$ y el efecto de la presión atmosférica $\left(\eta_{p}\right)$. Se construye usando la ecuación (8):

$$
\eta_{r}=\eta_{2}-\left(\eta_{m}+\eta_{p}\right)
$$

donde $\eta_{2}$ es el nivel del mar medido en Bahía Concepción, $\eta_{m}$ es la componente mareal del nivel del mar construida a partir de 8 componentes mareales (M2, K1, O1, Msf, S2, $\mathrm{N} 2, \mathrm{Q} 1, \mathrm{Mm})$ y $\eta_{p}$ es la componente de presión atmosférica construida con la ecuación hidrostática:

$$
\eta_{p_{0}}=-\frac{p-p_{0}}{\rho g}
$$

donde $p$ es la presión atmosférica instantánea (medida) y $p_{0}$ es una presión atmosférica de referencia del lugar (en este caso, $p_{0}=997.2 \mathrm{mbar}$ ). Los datos usados de nivel del $\operatorname{mar}\left(\eta_{2}\right)$, presión atmosférica instantánea $(p)$ y componente norte-sur del viento $\left(v_{y}\right)$ se muestran en la Tabla 3. Cabe notar que se utilizó un período de un año.

Tabla 3: Datos usados para cuantificar el efecto mecánico del viento en el forzante desnivel del mar. Columnas: variable, período, frecuencia $(\Delta t)$ y fuente de los datos: Servicio

\begin{tabular}{|c|c|c|c|}
\hline Variable (Figura 1) & Período & $\Delta t, \mathrm{~s}$ & Fuente \\
\hline$\eta_{2}$ & \multirow{3}{*}{$\begin{array}{l}1 / 01 / 2016 \text { a } \\
31 / 12 / 2016\end{array}$} & 60 & SHOA \\
\hline$p$ & & \multirow{2}{*}{3600} & \multirow{2}{*}{ INIA } \\
\hline $\mathrm{v}_{y}$ & & & \\
\hline
\end{tabular}
Hidrográfico y Oceanográfico de la Armada (SHOA) e Instituto de Investigaciones Agropecuarias de Chile (INIA).

\section{Volumen acumulado y compuerta}

El flujo de marea es en general simétrico con respecto a las fases de marea. Esto implica que el efecto mareal traería de vuelta a Bahía Concepción un volumen equivalente de agua al transportado hacia Bahía San Vicente en la fase contraria de la marea. Para evitar que agua de Bahía San Vicente ingrese a Bahía Concepción, se considera la instalación de una compuerta. Usar la compuerta implica que aproximadamente durante la mitad del tiempo no habría flujo de agua entre las bahías. La compuerta sería un sistema de dos portalones de eje vertical, sistema habitual de manejo de flujo en canales artificiales.

Con el transporte (ecuación (6)) se calcula el volumen acumulado en ambas bahías. En un segundo escenario, se calcula el volumen acumulado suponiendo que existe una compuerta mareal en el canal que permite el ingreso 
solamente hacia la Bahía San Vicente. Numéricamente, la compuerta existe cuando la velocidad del flujo es positiva ( $u>0$, es decir, cuando el agua se mueve hacia Bahía Concepción). En tales casos, se considera $U=0$.

\section{Resultados}

\section{Velocidad y transporte}

La velocidad del flujo $(u)$ y el transporte de volumen $(U)$ en el CIB durante tres días de sicigia y tres días de cuadratura son mostrados en la Figura 3. Se puede observar la evidente dominancia de la marea semidiurna (M2) en todas las curvas. En la Figura 3 también se observan oscilaciones de alta frecuencia. Un análisis espectral muestra que el principal período de esas perturbaciones es cercano a 1.7 horas. Muy probablemente esas oscilaciones correspondan al aporte de variaciones en el nivel del mar, en cada una de las dos bahías asociadas a oscilaciones propias (seiches). En Bahía Concepción, por ejemplo, la seiche fundamental tiene un período cercano a $95 \mathrm{~min}$, que corresponde a unas 1.6 horas (Aránguiz et al., 2014).

a)
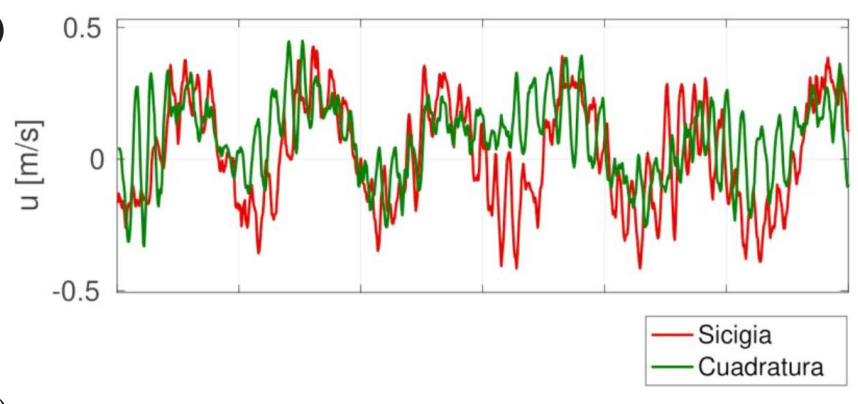

b)

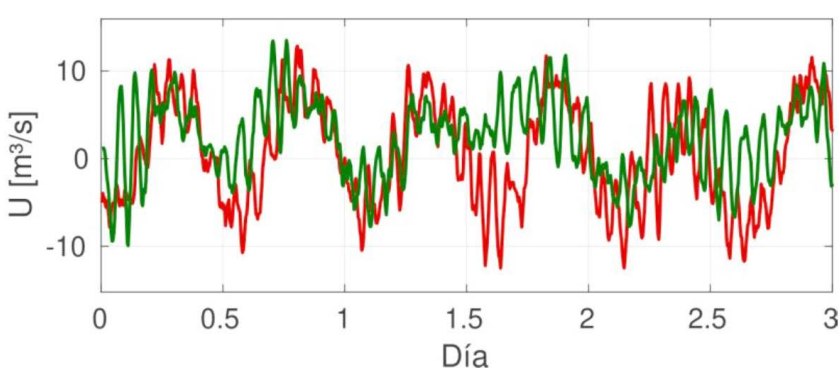

Figura 3: (a) Velocidad del flujo $(u)$ y $(b)$ Transporte de volumen $(U)$ en el Canal Interbahías para períodos de 3 días centrados en sicigia (curva roja) y cuadratura (curva verde)

Ahora, las curvas de sicigia y cuadratura son un tanto diferentes entre sí. Esta diferencia está explicada por el efecto del arrastre del viento. Esto se comprende mejor mirando la Tabla 4. De los tres forzantes, dos inducen el flujo: el desnivel del mar y el arrastre del viento. En la Tabla 4 se puede observar que el causante dominante del flujo es el desnivel del mar y en segundo lugar el arrastre del viento. El forzante roce de fondo reduce el flujo y representa un efecto importante. El aporte del desnivel del mar y del roce fueron construidos haciendo simulaciones independientes para cada caso. El aporte del arrastre del viento fue construido a partir de la diferencia entre la simulación con y sin el término asociado al viento.

Tabla 4: Contribución de cada forzante del flujo (marea, roce de fondo y arrastre del viento, sección Modelo numérico) a la velocidad en el CIB. Primera columna: forzante. Segunda columna: velocidad máxima $\left(u_{\max }\right)$ inducida por cada forzante. Tercera columna: magnitud promedio de la velocidad $(|\bar{u}|)$ inducida por cada forzante.

\begin{tabular}{|c|c|c|}
\hline Término & $u_{\max }, \mathrm{cm} / \mathrm{s}$ & $|\bar{u}|, \mathrm{cm} / \mathrm{s}$ \\
\hline Todos & 58.0 & 16.00 \\
\hline Desnivel & 63.6 & 16.00 \\
\hline Roce & 23.5 & 4.20 \\
\hline Viento & 7.5 & 0.33 \\
\hline
\end{tabular}

\section{Efecto acumulativo del viento}

La comparación del nivel del mar residual $\left(\eta_{\mathrm{r}}\right)$ y la componente norte-sur del viento $\left(v_{y}\right)$ se presenta a continuación. La máxima correlación de Pearson entre las dos series de tiempo (-0.72) ocurre con un desfase temporal de 5 horas. Esto sugiere que durante eventos de viento de 2 a 5 días, el viento debe soplar durante 5 horas para provocar un cambio en el nivel del mar de la Bahía Concepción. La relación entre ambas serie de tiempo se puede visualizar en la Figura 4. Dado el desfase de 5 horas entre ambas series, el nivel del mar residual ha sido desplazado 5 horas para observar más claramente la relación. En la Figura 4,

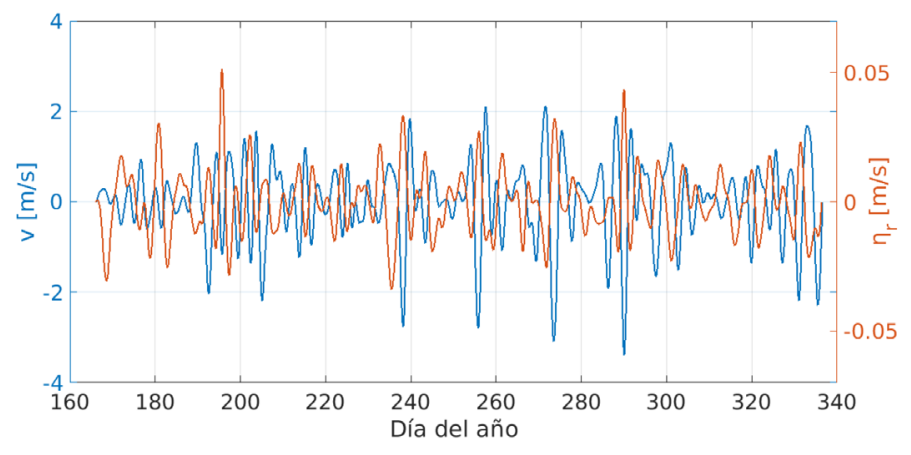

Figura 4: Componente v del viento en Bahía Concepción (curva azul) versus nivel del mar residual en Bahía Concepción (curva roja). El nivel del mar residual ha sido desplazado 5 horas considerando el desfase de 5 horas entre ambas series de tiempo. A ambas series de tiempo se les aplicó un filtro Coseno-Lanczos pasa-banda, rescatando frecuencias asociadas a períodos de entre 2 y 5 días. Se muestra un período de 6 meses y no de un año para poder apreciar mejor la figura. 
se ve claramente que cuando la componente v (norte-sur) del viento en Bahía Concepción es positivo (viento sur), el nivel del mar residual disminuye. Esto es lógico pues el viento saca agua de la bahía en este caso. Cuando la componente $\mathrm{v}$ del viento es negativa (viento norte), el agua se acumula en Bahía Concepción aumentando el nivel del mar residual. Cabe notar que ambas series de tiempo fueron filtradas para rescatar sólo los eventos de viento entre 2 a 5 días. El filtro utilizado fue un Coseno-Lanczos pasa-banda entre períodos de 48 y 120 horas.

Con un desfase temporal de 5 horas el cambio en el nivel del mar de Bahía Concepción que provoca el viento se puede expresar mediante una pendiente:

$$
c=\frac{0.0092}{-1 m / s}
$$

lo cual puede interpretarse como: un evento de entre 2 y 5 días de viento, con componente $v_{y}$ del viento en Punta de Parra provoca un aumento en el nivel del mar de $9.2 \mathrm{~mm}$, o sea, de casi un centímetro por cada $1 \mathrm{~m} / \mathrm{s}$, hacia al sur, de intensidad del viento. Este aporte no fue ingresado en el forzante desnivel del mar para producir la Figura 3. El análisis fue realizado aparte. La magnitud del aporte en la velocidad del efecto del viento vía cambio del nivel del mar tiene una magnitud promedio de $15 \mathrm{~cm} / \mathrm{s}$ (PedrerosGuarda, 2020), lo cual es relevante considerando que la velocidad en el canal es del orden de $\mathrm{cm} / \mathrm{s}$. Cabe notar que en general, sin considerar este efecto, la amplitud de la pérdida de carga en el CIB provocada por la componente mareal semidiurna M2 resulta ser de $0.013 \mathrm{~m}$. Ello produce velocidades promedio de $16 \mathrm{~cm} / \mathrm{s}$ (Tabla 4). Con esto queda de manifiesto que el CIB es bastante sensible al desnivel del mar.

\section{Volumen acumulado y compuerta}

La Figura 5 muestra el volumen de agua acumulado en las bahías Concepción y San Vicente en escenarios con y sin compuerta en el canal. Estos escenarios son en distintos eventos del ciclo lunar. Las fechas se muestran en la Tabla 5. En ambos casos el volumen acumulado por día en las bahías es del orden de $1 \times 10 \square \mathrm{m}^{3}$, equivalente a un cubo de agua de unos $46 \mathrm{~m}$ de arista.

Sin compuerta (Figura 5a) estos volúmenes pueden ser positivos (se acumulan en Bahía Concepción) o negativos (se acumulan en Bahía San Vicente). Pero la velocidad promedio es de $-0.6 \mathrm{~mm} / \mathrm{s}$. Esto indicaría que el flujo tiende a ir hacia Bahía San Vicente. Con compuerta (Figura 5b) naturalmente todos los volúmenes de agua son negativos, es decir, se acumulan en Bahía San Vicente (pues la compuerta bloquea el flujo hacia Bahía Concepción).

Tabla 5: Fecha y hora de los eventos del ciclo lunar analizados (hora local: UTC-4)

\begin{tabular}{|c|c|}
\hline Evento & Fecha \\
\hline Sicigia 1 (S1) & $28-04-2010,8: 18 \mathrm{hrs}$ \\
\hline Sicigia 2 $(\mathrm{S} 2)$ & $13-05-2010,21: 00 \mathrm{hrs}$ \\
\hline Cuadratura 1 $(\mathrm{C} 1)$ & $21-04-2010,14: 20 \mathrm{hrs}$ \\
\hline Cuadratura 2 (C2) & $06-05-2010,00: 15 \mathrm{hrs}$ \\
\hline
\end{tabular}

a)

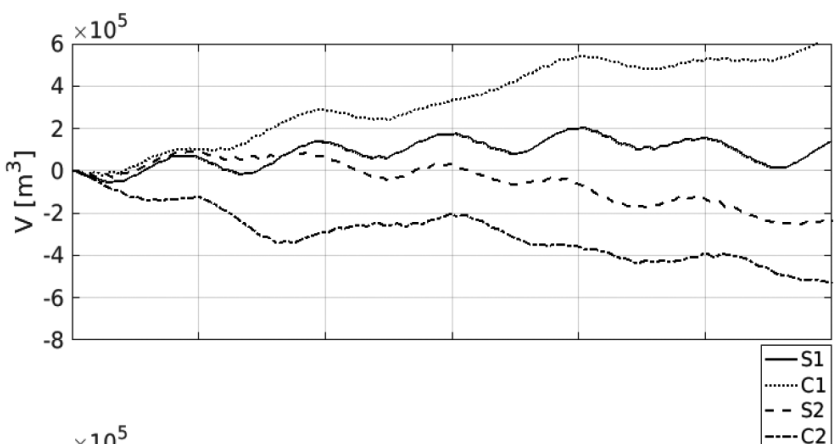

b)

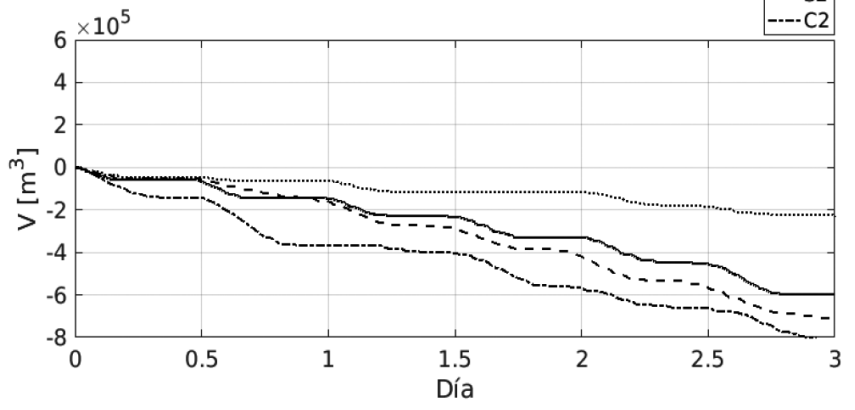

Figura 5: (a) Volumen acumulado sin compuerta y (b) con compuerta en el CIB para períodos de 3 días en distintos eventos de la fase lunar (curvas negras, ver Tabla 5)

\section{Renovación de las aguas en bahía San Vicente}

Para entender cómo influye el canal en la renovación de las aguas de la Bahía San Vicente, se realiza un ejercicio sencillo. Mirando la Figura 5 es razonable decir que en un ciclo mareal (medio día) se acumula un volumen de aproximadamente $100 \mathrm{mil} \mathrm{m}^{3}$ de agua en Bahía San Vicente. Considerando que el volumen de la bahía es de 278.4x10 $\mathrm{m}^{3}$ (Rudolph et al., 2002), y sin considerar circulación local, se tiene que la bahía se renueva completamente en aproximadamente 3.8 años, por el efecto del canal. Ahora, considerando solamente el volumen de los primeros $3 \mathrm{~m}$ 
de la columna de agua, que es donde el canal tendría mayor incidencia, debido a que es la profundidad del canal, este volumen de agua se renueva en alrededor de 8.4 meses. Este cálculo fue realizado asumiendo que el volumen de la bahía es el producto entre un área horizontal constante y una profundidad. Bahía San Vicente tiene $16 \mathrm{~m}$ de profundidad promedio (Rudolph et al., 2002). Luego, el volumen por cada metro de profundidad puede obtenerse según: $V_{\text {metro }}=$ $278.4 \times 10^{6} \mathrm{~m}^{3} / 16$. El volumen de los 3 primeros metros es: $V_{3 \text { metros }}=V_{\text {metro }} x 3$. Más específicamente, se podría considerar solamente el volumen de la zona norte, que es la más contaminada y donde el canal tiene la mayor incidencia, porque es el lugar en que el CIB empalma con Bahía San Vicente. En la sección Introducción se menciona que el sector de puerto e industrial comprende alrededor del 15\% de la superficie de la bahía. Debido al canal, los primeros 3 $\mathrm{m}$ de agua de esta zona se renovarían en tan solo 1.2 meses.

\section{Discusión}

El propósito principal de este trabajo es evaluar si es factible que las mareas y el viento transporten un volumen de agua significativo desde Bahía Concepción hacia Bahía San Vicente. Para ello se eligió la obra más sencilla posible, un canal abierto. Habiendo ahora probado que este canal cumpliría su objetivo, se puede pensar en siguientes etapas de este trabajo, que pueden considerar variantes tales como el uso de un túnel, en lugar de un canal abierto, o "tapar" la superficie al canal (en que por una parte se recupera el terreno y se evita el efecto del viento suroeste, pero por otra parte se impide la navegación en el canal), el trazado de un canal que no tenga un trazado recto, etc.

Atendiendo la obra propuesta (canal abierto), en este trabajo se plantea un estudio preliminar para renovar las aguas de la Bahía San Vicente, usando la energía de forzantes naturales. Es un estudio exclusivamente de los aspectos dinámicos, por lo que es natural que al pensar en una implementación real surjan una serie de preguntas que este trabajo no considera. Algunas de ellas, como si el canal podría inducir una inundación en la Bahía San Vicente, o qué pasa con el flujo del canal en ráfagas de viento, fueron respondidas en Pedreros-Guarda (2020). En ese mismo trabajo se realizó un test de consistencia del modelo, para el caso del Canal de Chacao, obteniéndose buenos resultados.
Otro aspecto no considerado aún es cómo afecta este canal a la circulación marina en Bahía San Vicente, ni tampoco las implicancias ambientales del canal. Ambos aspectos serán objeto de estudios adicionales. Sin embargo, es importante añadir de antemano que en este estudio se ha asumido que el ingreso de aguas hacia Bahía San Vicente mejorará su calidad de agua y no al revés. Por eso, de implementarse algo así, sería conveniente que vaya de la mano con un sistema de monitoreo de la calidad del agua del sistema de bahías San Vicente y Concepción. Hoy, existen numerosas técnicas de teledetección para obtener campos espaciales de parámetros de calidad del agua en el océano (Gholizadeh et al., 2016). Incidentalmente, se puede mencionar que ya existió un "Canal Interbahías", dado que la zona del CIB ya estuvo inundada hace 6000 años (Link et al., 2019).

\section{Conclusiones}

Si dos cuerpos de agua unidos por un canal tienen distinto nivel del mar, un flujo es inducido en el canal. El flujo en el Canal Interbahías propuesto es modulado principalmente por el desnivel del mar y en segundo lugar por el arrastre del viento. El desnivel del mar es causado por la onda de marea y por el efecto del viento en la acumulación o extracción de agua de la Bahía Concepción. Cuando la intensidad del viento es baja y la dirección variable, el forzante dominante del desnivel del mar es la onda de marea. Pero cuando la intensidad del viento es alta y su dirección constante, el efecto acumulativo del viento y la onda de marea contribuyen en magnitud similar al desnivel del mar.

Finalmente, se concluye que físicamente el Canal sí aportaría a la renovación de las aguas de la Bahía San Vicente. Además, los vientos típicos de la zona producirían una renovación de las aguas de la parte más crítica de bahía San Vicente del orden de un mes, si es que el canal opera con una compuerta.

\section{Agradecimientos}

Agradecemos al Servicio Hidrográfico y Oceanográfico de la Armada por proporcionar los datos del nivel del mar que hicieron posible este trabajo. Agradecemos a Marcus Sobarzo y al Sistema de Información Nacional de Calidad del Aire por proporcionar datos de viento. También a Freddy Echeverría y Cristian Salas por colaborar con 
datos necesarios en el proceso. Finalmente a Carolina Parada y Martín Jacques por valiosas sugerencias durante la realización de este estudio.

\section{Referencias}

Aguirre-Martínez, G., Rudolph, A., Ahumada, R., Loyola, R. y Medina, V. (2009). Toxicidad no específica en sedimentos portuarios, una aproximación al contenido de contaminantes críticos. Revista de Biología Marina y Oceanografía 44(3), 725735

Ahumada, R., Rudolph, A., Madariaga, S. y Carrasco, F. (1989). Descripción de las condiciones oceanográficas de Bahía San Vicente y antecedentes sobre los efectos de la contaminación. Biología Pesquera 18, 37-52

Ahumada, R. (1992). Patrones de distribución espacial de Cr, $\mathrm{Ni}, \mathrm{Cu}, \mathrm{Zn}, \mathrm{Cd}$ y $\mathrm{Pb}$, en sedimentos superficiales de bahía San Vicente, Chile. Revista de Biología Marina, Valparaíso 27(2), 265-281

Ahumada, R., Rudolph, A. and Martinez, V. (1983). Circulation and fertility of waters in Concepción Bay. Estuarine, Coastal and Shelf Science 16(1), 95-105

Aránguiz, R., Villagrán, M., Esteban, M. and Shibayama, T. (2014). Tsunami resonance in the Bay of Concepcion, Chile. Proceedings of the $34^{\text {th }}$ Conference on Coastal Engineering, Seoul, South Korea

Blanchfield, J., Garrett, C., Wild, P. and Rowe, A. (2008). The extractable power from a channel linking a bay to the open ocean. Proceedings of the Institution of Mechanical Engineers, Part A: Journal of Power and Energy 222(3), 289-297

Chow, V.T. (1994). Hidráulica de canales abiertos. $4^{\text {ta }}$ ed., McGraw-Hill, México

DIRECTEMAR (2019). Boletín estadístico marítimo. Armada de Chile, Valparaíso, Chile

Falvey, M. and Garreaud, R. (2007). Wintertime precipitation episodes in central Chile: Associated meteorological conditions and orographic influences. Journal of Hydrometeorology 8(2), 171-193

Garrett, C. and Cummins, P. (2005). The power potential of tidal currents in channels. Proceedings of the Royal Society A: Mathematical, Physical and Engineering Sciences 461(2060), 2563-2572
Gholizadeh, M.H., Melesse, A.M. and Reddi, L. (2016). A comprehensive review on water quality parameters estimation using remote sensing techniques. Sensors 16(8), 1298.

Link, O., Brox-Escudero, L.M., González, J., Aguayo, M., Torrejón, F., Montalva, G. and Eguibar-Galán, M.Á. (2019). A paleo-hydro-geomorphological perspective on urban flood risk assessment. Hydrological Processes 33(25), 3169-3183

Pedreros-Guarda, M. (2020). Estudio físico de un canal Interbahías para la renovación de las aguas en la Bahía San Vicente, Región del Biobio, Chile. Título profesional de Geofísica, Universidad de Concepción, Chile

Rudolph, A., Ahumada, R. and Pérez, C. (2002). Dissolved Oxygen content as an index of water quality in San Vicente Bay, Chile (36 $\left.{ }^{\circ \prime} \mathrm{S}\right)$. Environmental Monitoring and Assessment 78(1), $89-100$

Russell, T.F. (1989). Stability analysis and switching criteria for adaptive implicit methods based on the CFL condition. Symposium on Reservoir Simulation, SPE, Houston, USA, $97-$ 107

Sobarzo, M.B., Figueroa, D. and Arcos, D.R. (1997). The influence of winds and tides in the formation of circulation layers in a bay, a case study: Concepción Bay, Chile. Estuarine, Coastal and Shelf Science 45(6), 729-736

Solorza, S., Yee-Romero, C., Jordan-Aramburo, A. and CardeñaSánchez, S. (2010). El término de error en los esquemas de diferencias finitas. Latin-American Journal of Physics Education 4(1), 168-173

Srain, B. and Rudolph, A. (2010). Acetylcholinesterase activity, antioxidant defenses, and lipid peroxidation in the clam Semele solida: Can this species be used as a bioindicator?. Revista de Biología Marina y Oceanografía 45(2), 227-233

Trenberth, K.E., Large, W.G. and Olson, J.G. (1989). The effective drag coefficient for evaluating wind stress over the oceans. Journal of Climate 2(12), 1507-1516

Valenzuela, J. (2003). Caso: Recuperación ambiental integral de la Comuna de Talcahuano, Chile. Asociación Mundial del Agua, Estocolmo, Suecia

Vallis, G.K. (2006). Atmospheric and oceanic fluid dynamics: fundamentals and large-scale circulation. Cambridge University Press, UK, 107, 702-703 


\section{Pedreros-Guarda, M. y Figueroa, D. (2021). Obras y Proyectos 30, 6-15}

Vennell, R. (2010). Tuning turbines in a tidal channel. Journal of Fluid Mechanics 663, 253-267

Vennell, R. (2011). Estimating the power potential of tidal currents and the impact of power extraction on flow speeds. Renewable Energy 36(12), 3558-3565

Wu, F.C., Shen, H.W. and Chou, Y.J. (1999). Variation of roughness coefficients for unsubmerged and submerged vegetation. Journal of Hydraulic Engineering 125(9), 934-942 